\title{
Ballistic Phonons in Ultrathin Nanowires
}

\author{
Daniel Vakulov, ${ }^{\perp}$ Subash Gireesan, ${ }^{\perp}$ Milo Y. Swinkels, ${ }^{\perp}$ Ruben Chavez, Tom Vogelaar, Pol Torres, \\ Alessio Campo, Marta De Luca, Marcel A. Verheijen, Sebastian Koelling, Luca Gagliano, \\ Jos E. M. Haverkort, F. Xavier Alvarez, Peter A. Bobbert, Ilaria Zardo, and Erik P. A. M. Bakkers*
}

Cite This: Nano Lett. 2020, 20, 2703-2709

Read Online

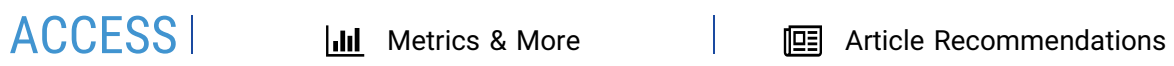

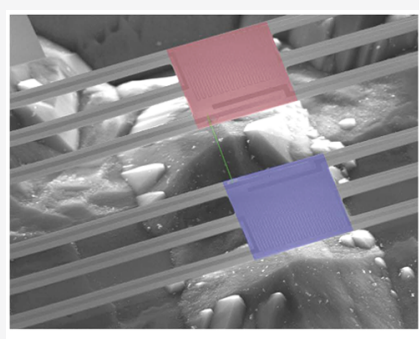

Supporting Information

ABSTRACT: According to Fourier's law, a temperature difference across a material results in a linear temperature profile and a thermal conductance that decreases inversely proportional to the system length. These are the hallmarks of diffusive heat flow. Here, we report heat flow in ultrathin $(25 \mathrm{~nm}) \mathrm{GaP}$ nanowires in the absence of a temperature gradient within the wire and find that the heat conductance is independent of wire length. These observations deviate from Fourier's law and are direct proof of ballistic heat flow, persisting for wire lengths up to at least $15 \mu \mathrm{m}$ at room temperature. When doubling the wire diameter, a remarkably sudden transition to diffusive heat flow is observed. The ballistic heat flow in the ultrathin wires can be modeled within Landauer's formalism by ballistic phonons with an extraordinarily long mean free path.

KEYWORDS: Nanowires, GaP, heat transport, ballistic phonons, Raman spectroscopy

$\mathrm{H}$ eat flow in macroscopic systems is traditionally described by Fourier's law for diffusive heat flow. ${ }^{1}$ The advancement of micro and nanotechnology has led in the past few years to observations of nondiffusive heat flow. Deviations from diffusive heat flow can occur due to the wave nature of phonons, ${ }^{2}$ hydrodynamic effects, ${ }^{3-10}$ and ballistic behavior. $^{11-18}$ In nanowires (NWs), scattering of phonons at the NW surface reduces the thermal conductivity as compared to the bulk. ${ }^{19}$ However, when the surface is atomically flat, phonons may reflect specularly from the surface ${ }^{20,21}$ and ballistic heat flow in the axial direction may occur.

Realizing ballistic flow over large distances at room temperature requires a long phonon mean free path (MFP), which is challenging due to different scattering mechanisms. Only a few studies have reported the observation of ballistic transport at room temperature. Ballistic transport at room temperature up to a length of $260 \mathrm{~nm}$ was reported in short and wide graphene ribbons. ${ }^{12}$ The heat transport is diffusive in short and narrow ribbons due to the presence of edge disorder. Room-temperature ballistic heat flow up to a length of $8.3 \mu \mathrm{m}$ was reported for SiGe NWs with diameters in the range 50$200 \mathrm{~nm} .{ }^{13}$ Its occurrence was attributed to the filtering out of high-frequency short-MFP phonons by alloy scattering. Beyond $8.3 \mu \mathrm{m}$, the heat flow in these NWs was found to be diffusive. Ballistic phonon transport at room temperature was also reported in holey silicon up to a length of $200 \mathrm{~nm} .{ }^{15}$ The explanation in this case was that surface disorder filters out high-frequency phonons, leaving the ballistic transport of lowfrequency phonons.
In this letter, we demonstrate room-temperature axial ballistic heat flow in ultrathin, $25 \mathrm{~nm}$ diameter, GaP NWs up to wire lengths of at least $15 \mu \mathrm{m}$, and a sharp transition to diffusive heat flow when the diameter is doubled to $50 \mathrm{~nm}$. Room-temperature ballistic transport over such large distances and a diffusive-ballistic transition with decreasing lateral dimension have not been reported thus far. The temperature profile along the NWs is obtained by Raman thermometry, confirming the presence of a temperature gradient in the diffusive flow regime and its absence in the ballistic regime.

We grow GaP NWs with 25-140 nm diameters by metalorganic vapor-phase epitaxy using the vapor-liquid-solid growth technique (see Supplementary Section SI1). The NWs are grown in arrays by substrate/catalyst patterning. Within an array, the NWs have a uniform diameter along their length with a $<5 \mathrm{~nm}$ diameter spread. We analyze the structural properties of the NWs by transmission electron microscopy (TEM). TEM images show stacking-fault-free NWs, grown along the $\langle 0001\rangle$ direction (see Figure 1a). The NWs have atomically flat $\{11 \overline{0} 0\}$ side facets covered by an approximately $2 \mathrm{~nm}$-thick amorphous oxide layer (see Figure 1b). Electron diffraction patterns taken in the TEM further show that NWs

Received: January 23, 2020

Published: February 24, 2020 

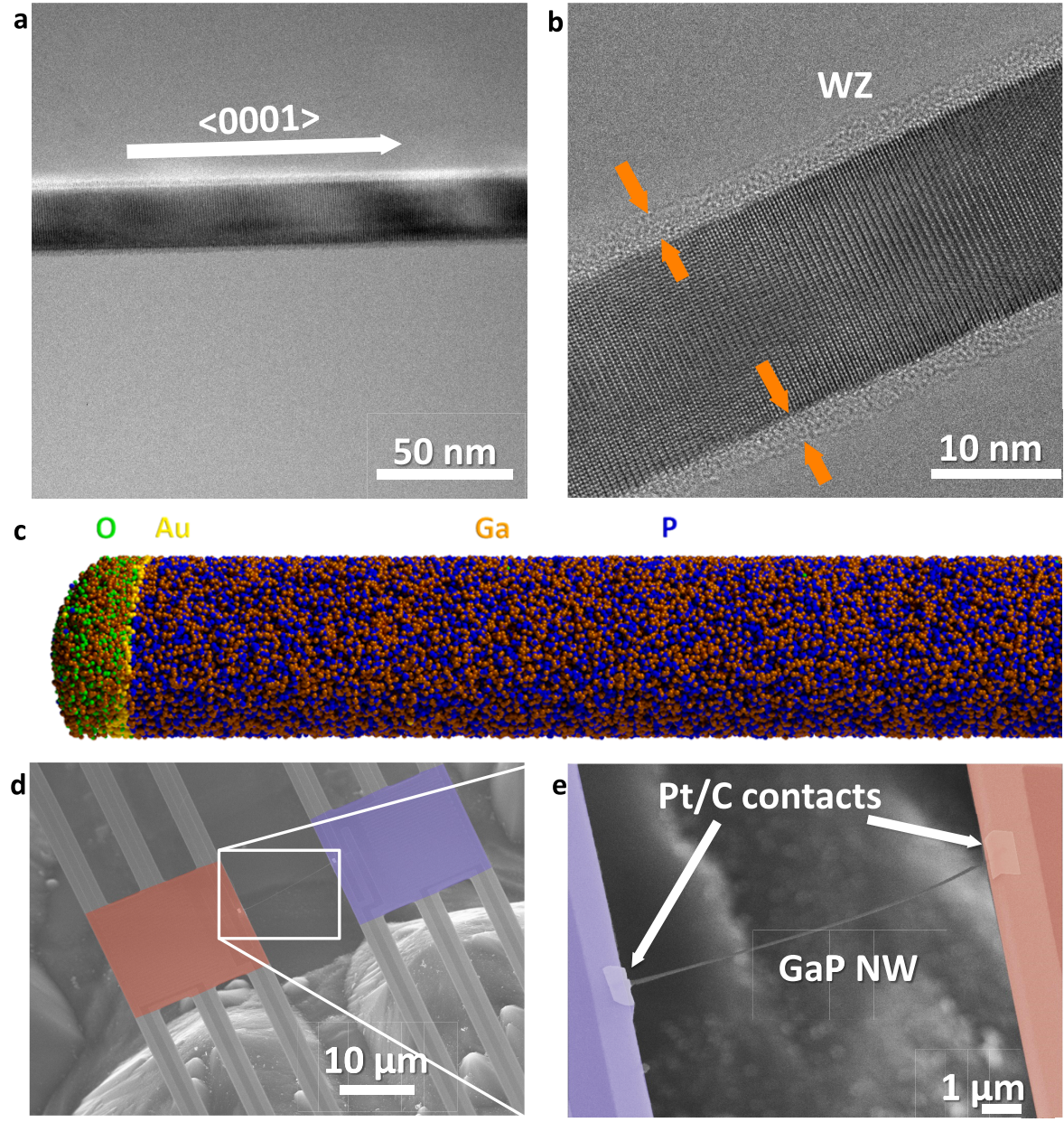

Figure 1. GaP NW properties and device for thermal conductance measurements. (a) TEM image of a 25 nm diameter GaP NW, grown along the $\langle 0001\rangle$ direction. (b) High-resolution TEM image taken along the $\langle 11 \overline{2} 0\rangle$ direction of a NW, showing a defect-free WZ crystal structure and a layer of oxide (orange arrows). (c) APT analysis of a $25 \mathrm{~nm}$ diameter NW showing its high chemical purity. (d) $45^{\circ}$-tilted SEM image of the thermal conductance measuring device, with suspended heating (red) and sensing (blue) membranes. (e) Zoom-in of the GaP NW bridging the suspended membranes. Electron beam-induced deposited $\mathrm{Pt} / \mathrm{C}$ improves the thermal contact.

with different diameters used in our study all have a wurtzite (WZ) crystal structure (see Figure S1). Atom-probe tomography (APT) demonstrates the chemical purity of the NWs and the absence of heavy element impurities (see Figures 1c and S2). The NWs are therefore atomically clean and defect-free. Our GaP NWs are thus ideal systems to look for ballistic heat transport. Limitations of the APT technique prevent detection of the oxide layer. ${ }^{22}$ We fabricate microdevices $^{23,24}$ to study the thermal conductivity of individual NWs (see Section SI3). The devices consist of two suspended membranes with $\mathrm{Pt}$ meander structures on top, which can be used as heater and thermometer; see the scanning electron microscopy (SEM) picture in Figure 1d. Different spacings between the membranes allow measuring NWs of different lengths. Using a micromanipulator under an optical microscope, we place as-grown single NWs with selected diameter between the membranes. $\mathrm{Pt} / \mathrm{C}$ contacts are deposited on top of the NWs to improve the thermal contact (see Figure 1e). We determine the thermal conductivity $\kappa$ from the measured thermal conductance $G$ and the NW dimensions. The thermal conductance of the NW, which is defined as the amount of heat flux going through a material when one Kelvin temperature difference is applied, is determined by applying a current to one of the coils and measuring the temperature on both sides of the NW. From these measurements, the NW thermal conductance is extracted through a heat balance calculation for the sensing membrane, where the heat transported through the wire is put equal to the heat that leaves the membrane through the $\mathrm{SiN}_{x}$ beams, as is commonly done when employing this kind of devices (see Section SI3 and ref 23.).

The extracted $\kappa$ at room temperature $T=300 \mathrm{~K}$ is plotted in Figure $2 \mathrm{a}$ as a function of diameter $d$ for NWs with approximately the same length $L=6.7 \pm 0.1 \mu \mathrm{m}$, with $d$ ranging from 25 to $140 \mathrm{~nm}$. These results were fitted to expose the influence of the thermal contact resistance, $R_{c}{ }^{24}$ In a NW with heat flow dominated by diffuse scattering at the NW surface, $\kappa$ increases with $d$ according to Mathiessen's rule $\kappa \propto$ $\left(1+l_{\text {bulk }} / d\right)^{-1}$, where $l_{\text {bulk }}$ is the bulk MFP, as indicated by the dashed line. A reduced $\kappa$ with respect to this line, which is observed for $d \geq 75 \mathrm{~nm}$, indicates that $R_{\mathrm{c}}$ is no longer negligible compared to the NW resistance. ${ }^{24}$ For thin wires $(d$ $<50 \mathrm{~nm}$ ), on the contrary, an enhanced $\kappa$ is observed, indicating that a nondiffusive transport mechanism, not dominated by a contact resistance, becomes progressively important with decreasing diameter. To investigate the 
a

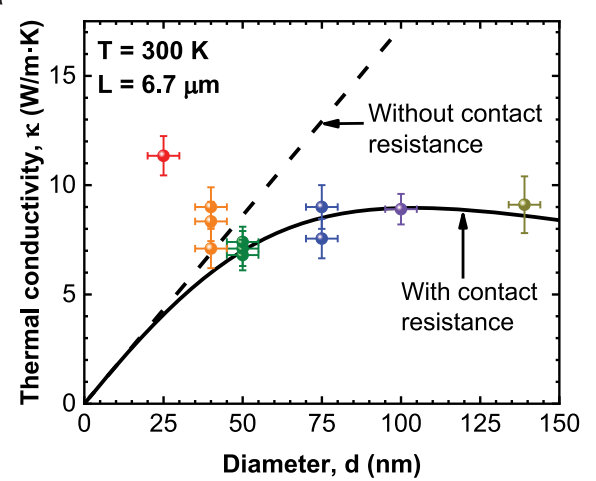

C

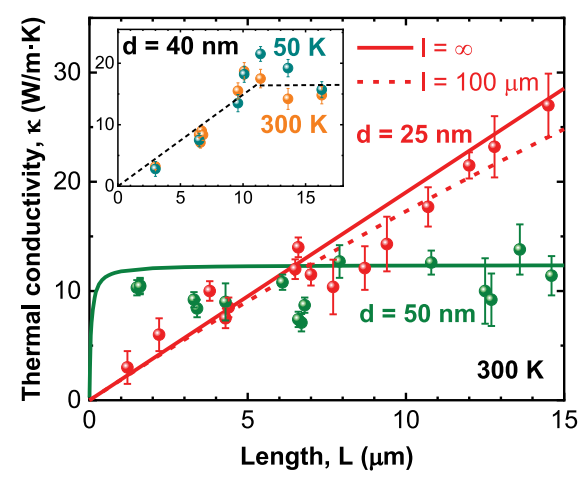

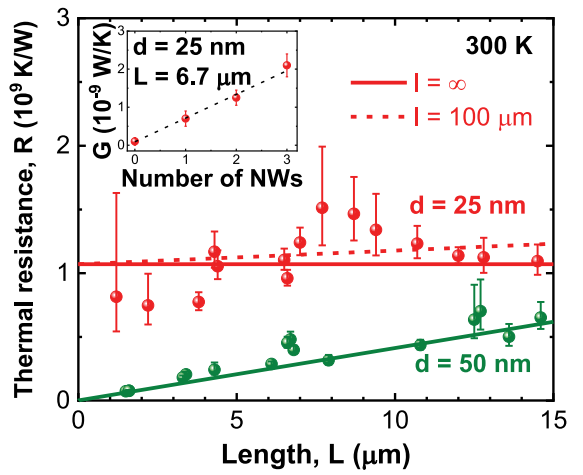

d

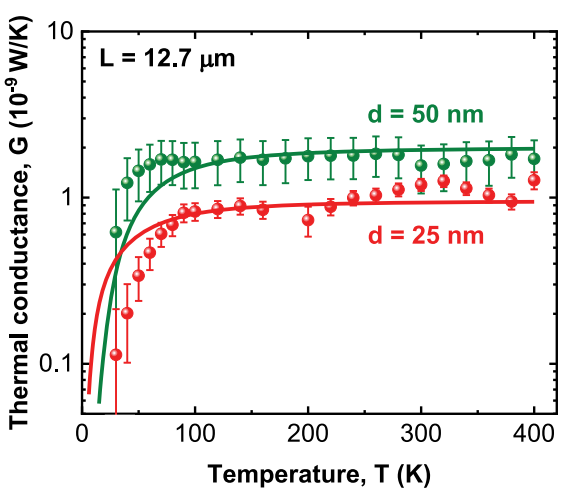

Figure 2. Transition from diffusive to ballistic heat flow. (a) Room-temperature $(300 \mathrm{~K})$ thermal conductivity $\kappa$ of GaP NWs of length $L=6.7 \pm$ $0.1 \mu \mathrm{m}$ as a function of diameter $d$. Full curve: Fit of results for $d \geq 50 \mathrm{~nm}$ to $\kappa=\left(1 / c_{1} d+c_{2} \pi d / 4 L\right)^{-1}$ (diffusive heat flow with MFP $l=d \ll l_{\text {bulk }}$ and contact resistance $R_{c}=c_{2} / d$, with $c_{1}=1.7 \times 10^{8} \mathrm{~W} / \mathrm{m}^{2} \cdot \mathrm{K}$ and $c_{2}=4.6 \mathrm{~m} \cdot \mathrm{K} / \mathrm{W}$ ). Dashed line: $\kappa=c_{1} d$ (diffusive heat flow without contact resistance and $d \ll l_{\text {bulk }}$ ). (b) Symbols: Measured length dependence of resistance $R$ of NWs with $d=25$ and 50 nm. Lines: Modeling results based on Landauer's formalism, using the numbers of specular phonon modes $N_{\mathrm{s}}(d=25 \mathrm{~nm}$, solid line: MFP $l=\infty$, dashed line: $l=100 \mu \mathrm{m})$ and diffusive phonon modes $N_{\mathrm{d}}(d=50 \mathrm{~nm}, \mathrm{MFP} l=d)$ from Figure $S 12$. For $d=25 \mathrm{~nm}$, the curve for $l=100 \mu \mathrm{m}$ is obtained by dividing the result for $l=\infty$ by the transmission coefficient $l /(l+L) .{ }^{30}$ Inset: Conductance $G$ of a device without a NW (see Section SI4) and with 1,2 , and 3 NWs with $d=25 \mathrm{~nm}$ and $L=6.7 \pm 0.1 \mu \mathrm{m}$. Dashed line: Linear fit. (c) Same results as in main panel of (b), but for the conductivity $\kappa$. Inset: Results for $d=$ $40 \mathrm{~nm}$ NWs measured at 300 and $50 \mathrm{~K}$ (measurements on different devices at the different temperatures). Dashed line: Guide to the eye. (d) Measured (symbols) and modeled (curves) T-dependent thermal conductance $G$ of $d=25$ and $50 \mathrm{~nm}$ NWs with length $L=12.7 \pm 0.2 \mu$ m.

transport mechanism in the thin wires, the thermal resistance $R$ $=1 / G$ has been plotted as a function of NW length $L$ for diameters $d=25$ and $50 \mathrm{~nm}$ in Figure $2 \mathrm{~b}$ (main panel). For the $50 \mathrm{~nm}$ NWs, $R$ is linearly dependent on $L$, in accordance with diffusive heat flow. For the $25 \mathrm{~nm}$ NWs, however, $R$ is independent of $L$ for all measured NWs up to $L=15 \mu \mathrm{m}$, which is proof of ballistic transport. We attribute this resistance to a small number of ballistic modes contributing to transport (see below). We note that the extrapolation of the $50 \mathrm{~nm}$ data goes through the origin, corroborating a negligible $R_{\mathcal{O}}$ in agreement with a model where $R_{\mathrm{c}}$ is taken inversely proportional to the contact area between contact and NW (solid curve in Figure 2a). Although we cannot estimate $R_{\mathrm{c}}$ for the $25 \mathrm{~nm}$ NWs directly from the measurements, a negligible contact resistance follows from this model. The measured linear dependence of the device conduction with the number of NWs for $d=25 \mathrm{~nm}$ and $L=6.7 \pm 0.1 \mu \mathrm{m}$ (inset in Figure $2 \mathrm{~b}$ ) excludes the influence of a possible background conduction. We note that these data have been collected from a device with a gap between the $\mathrm{SiN}_{x}$ membranes of 4 $\mu \mathrm{m}$, where we put down the NWs under an angle to measure NWs with $L=6.7 \pm 0.1 \mu \mathrm{m}$. We have also characterized devices with a smaller gap size of $2 \mu \mathrm{m}$. Also for these devices, the influence of a possible background conduction can be excluded. The data therefore unequivocally point at a transition from diffusive to length-independent ballistic heat flow in $25 \mathrm{~nm}$ diameter NWs over unprecedented distances.

To further investigate the features of the diffusive-to-ballistic transition, we plot in Figure 2c (main panel) $\kappa v s L$ for $d=25$ and $50 \mathrm{~nm}$ NWs at $300 \mathrm{~K}$, clearly showing the remarkable fact that for long wires, the thermal conductivity of the ultrathin 25 $\mathrm{nm}$ NWs exceeds that of the thicker $50 \mathrm{~nm}$ NWs. The inset displays results for $40 \mathrm{~nm} \mathrm{NWs}$ at 300 and $50 \mathrm{~K}$, showing that at both room and low temperature, this is a transition case: The heat flow is ballistic up to $L \approx 11 \mu \mathrm{m}$ and then becomes diffusive. Figure $2 \mathrm{~d}$ shows a very weak $T$ dependence of the thermal conductance $G$ for both 25 and $50 \mathrm{~nm}$ NWs of $12.7 \pm$ $0.2 \mu \mathrm{m}$ length down to about $100 \mathrm{~K}$ (below this temperature, $G$ steeply decreases due to phonon freeze-out). Results for 75 $\mathrm{nm}$ NWs are comparable to those for $50 \mathrm{~nm}$ NWs, showing also a very weakly $T$-dependent diffusive heat flow (see Figure S6). The $T$ insensitivity rules out explanations for the diffusiveto-ballistic transition based on phonon-phonon interactions, which are strongly $T$ dependent. In particular, Umklapp scattering, which leads to a strong decrease of $\kappa$ in bulk GaP above $50 \mathrm{~K},{ }^{25}$ appears to play no significant role. Also, the involvement of phonon-polaritons as heat conducting species is unlikely, because they would yield a contribution to $G$ that is linear in $T .{ }^{26}$ The thermal conductivities shown in Figure 2 are significantly smaller than the room-temperature thermal 
conductivity of bulk GaP. For bulk GaP, experimental thermal conductivities are only available for the equilibrium zinc blende structure, with a room-temperature value of $75.2 \mathrm{~W} / \mathrm{m} \cdot \mathrm{K}^{27}$ The calculated bulk thermal conductivity of $\mathrm{WZ} \mathrm{GaP}$ is $92 \pm 5$ $\mathrm{W} / \mathrm{m} \cdot \mathrm{K} .^{28}$

To substantiate the ballistic transport in the thin NWs, we probe the thermal profile of 25 and $50 \mathrm{~nm}$ NWs with Raman thermometry, which is a contactless method, in the same devices as used for the thermal conductivity measurements (see Figure 3a). Here, the local average lattice temperature is

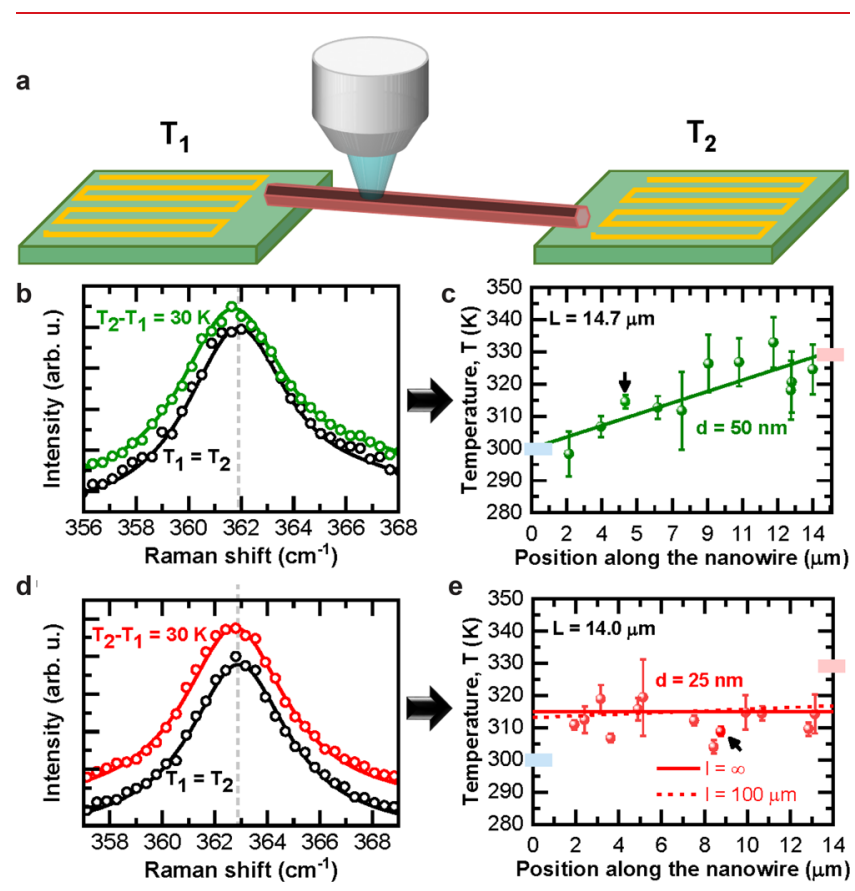

Figure 3. Temperature profiles in the diffusive and ballistic regimes. (a) Illustration of the microdevice with NW used for the measurements of the thermal profile. A laser spot is moved along the wire, and Raman spectra are acquired for $T_{1}=T_{2}=300 \mathrm{~K}$ and $T_{1}$ $=300 \mathrm{~K}, T_{2}=300 \mathrm{~K}+\Delta T$. (b) Spectra measured at the position indicated by the arrow in (c) for a NW with $d=50 \mathrm{~nm}$ and $L=14.7$ $\pm 0.3 \mu \mathrm{m}$. Solid lines: Lorentzian fits to the spectra. (c) Resulting temperature profile along the NW while applying $\Delta T=30 \mathrm{~K}$ using the $\mathrm{Pt}$ meander heaters. Solid line: Modeled profile for purely diffusive heat flow. (d) and (e) The same as (b) and (c) but for a NW with $d=25 \mathrm{~nm}$ and $L=14.0 \pm 0.3 \mu \mathrm{m}$. Solid line in (e): Modeled profile for purely ballistic transport with MFP $l=\infty$. Dashed line: Profile for $l=100 \mu \mathrm{m}$ with a transmission coefficient $l /(l+L)$, having a slope $[1-l /(l+L)] \Delta T / L .^{30}$ The bars at the left and right $y$-axis in (c) and (e) show the temperature of the contacts.

measured using the Raman shift of the transverse optical (TO) mode $^{29}$ (see Section SI6 for experimental details). We keep the system temperature at $T=300 \mathrm{~K}$ and heat the hot contact by $\Delta T=30 \mathrm{~K}$. Swapping the contacts yields the same results (see Figure S7). The frequency shift, $\delta \omega$, of the TO mode (at about $363 \mathrm{~cm}^{-1}$ in $\mathrm{GaP}$ ) with respect to the case without applying a temperature difference provides an estimate of the local lattice temperature increase, $\delta \mathrm{T}$, since $\delta \omega \propto \delta \mathrm{T}$ (see Figure $3 \mathrm{~b}, \mathrm{~d}$ ). This differential measurement cancels out changes in the TO mode frequency along the wire caused by other effects than the temperature difference, such as small laser heating effects and local strains. It is worth highlighting here that in the case of Stokes scattering (as we are employing), the measured spectrum reflects the average temperature of all phonon modes, since the frequency shift of the Raman peak is the result of higher-order phonon effects and thus involves all phonon modes. In other words, our Raman experiments measure an average lattice temperature. The temperature profile along the $50 \mathrm{~nm} \mathrm{NW}$ (Figure 3c) is linear, in agreement with diffusive transport. Importantly, the Raman thermometry measurements on the $50 \mathrm{~nm} \mathrm{NW}$ further confirm the absence of a significant contact resistance, since the measured temperature gradient agrees with the applied temperature difference, which was calibrated in an independent manner. In the $25 \mathrm{~nm} \mathrm{NW}$ (Figure 3e), however, we observe symmetric temperature jumps at the contacts and a constant temperature along the whole wire with $\delta T$ close to $15 \mathrm{~K}$, exactly as predicted by ballistic heat transport theory. ${ }^{30}$ We note that the TO mode itself contributes negligibly to the heat flow because of its very short MFP, but probes the average temperature of the heat-carrying phonons. In ballistic heat flow, heat-carrying phonons traveling in either direction through the NW have the temperature of the contact from which they were injected. The lattice thermalizes equally with the "hot" and "cold" phonons, and therefore, the TO mode probes the average of the contact temperatures, that is, $\delta T=15 \mathrm{~K}$. These results demonstrate the analogy with electrical transport through a ballistic quantum wire, where the electrical potential along the wire is constant and equal to the average of the potentials applied at the contacts. $^{31}$ To the best of our knowledge, this is the first measurement of the theoretically predicted temperature profile for ballistic heat transport. Together with the heat transport measurements of Figure 2, this provides strong experimental proof for ballistic heat flow. Interestingly, these measurements hint at the existence of a residual scattering between heatcarrying and non heat-carrying phonons.

We are not aware of an existing model in the literature that can capture a diffusive-to-ballistic transition for decreasing dimensions with the length scales involved in this work. To give a tentative explanation, we apply a model based on Landauer's formalism for phonon transport. It neglects anharmonic effects that lead to phonon-phonon scattering, in accordance with the weak $T$ dependence of our experimental data (see insets of Figure $2 c, d$ ). The model we apply has been developed by Murphy and Moore ${ }^{20}$ and Chen et al. ${ }^{32}$ (see Section SI7) and was successfully used to describe heat flow in silicon NWs ${ }^{32}$ and holey silicon. ${ }^{15}$ It distinguishes between two different types of phonon scattering at the NW surface: The surface appears sharp (diffuse) to phonons with an inverse perpendicular wave vector component longer (shorter) than the surface oxide layer thickness, leading to mostly specular (diffuse) scattering with long (short) MFP. $^{20,32}$ This description is also consistent with Lévy walk dynamics. ${ }^{18}$ We obtain the numbers of modes $N_{\mathrm{s}}$ and $N_{\mathrm{d}}$ of the "specular" and "diffusive" phonons at each frequency from $a b$ initio calculations of the phonon band structure of WZ GaP and phonon subband formation in the NW (see Section SI8). In Figure S12, these numbers are plotted for $d=25$ and $50 \mathrm{~nm}$ and an oxide layer thickness $h=2.45 \mathrm{~nm}$ (fitted to obtain agreement with the $d=25 \mathrm{~nm}$ thermal transport data, see below). This value of $h$ is close to the measured thickness (see Figure $1 \mathrm{~b}$ ). We note that the number of specular phonon modes $N_{\mathrm{s}}$ is only a small fraction $(\sim 1 \%)$ of the number of diffusive modes $N_{\mathrm{d}}$. We only consider phonons with a frequency below the Debye frequency. Higher-frequency phonons are hardly excited at the considered temperatures 
and have too short MFPs to significantly contribute to the heat flow.

A model where both contributions of specular phonons with a strongly increased MFP and of diffusive phonons with an MFP $l=d$ are considered equally does not agree with our data (see Figure S13). The contribution of diffusive phonons in the $25 \mathrm{~nm}$ NWs would lead to an offset in the thermal conductivity $\kappa$, and the contribution of specular phonons in the $50 \mathrm{~nm} \mathrm{NWs}$ would lead to a linear increase in $\kappa$, neither of which is observed. A suppression of the contribution to the heat flow of diffusive phonons in the $25 \mathrm{~nm}$ NWs accompanied by an enhancement of the contribution of specular phonons is in our model a necessary ingredient to explain the experimental data. As shown by the solid lines in Figure $2 b-d$, we obtain agreement with experiment both for 25 and $50 \mathrm{~nm}$ NWs if we: (1) neglect for the $50 \mathrm{~nm}$ NWs the heat flow carried by specular phonons in Landauer's expression for $G$ (eq S4 in Section SI7) and (2) disregard for the $25 \mathrm{~nm}$ NWs the contribution of diffusive phonons, taking only into account the heat flow carried by specular phonons with strongly increased MFP. A finite but strongly increased MFP $l$ up to $100 \mu \mathrm{m}$ would still agree with the heat transport data (dashed lines in Figure $2 b, c$ ) and would result in a small temperature gradient in the $25 \mathrm{~nm} \mathrm{NW}$ that is within the experimental uncertainty of the Raman measurements (dashed line in Figure 3e). Phonons in the $25 \mathrm{~nm}$ NWs therefore travel practically unimpeded and can truly be called ballistic. The weak $T$ dependence of the heat transport shown in Figure $2 \mathrm{~d}$ is explained by the fact that the main features in both $N_{\mathrm{s}}$ and $N_{\mathrm{d}}$ appear at phonon energies significantly below the thermal energy (see Figure S12). Although the original model of Murphy and Moore ${ }^{20}$ and Chen et al. $^{32}$ makes a distinction between specular and diffusive phonons, it contains no mechanism for the suppression of the heat flow carried by diffusive phonons and the emergence of ballistic character of the specular phonons. In their model, specular phonons still undergo a weak scattering into diffusive phonon modes caused by the disordered NW surface, preventing ballisticity.

A possible cause for the suppression of the heat flow carried by diffusive phonons is Anderson localization ${ }^{33}$ of these phonons in the $25 \mathrm{~nm}$ NWs. The phonon mean free paths of the diffusive phonons in the bulk WZ GaP crystal are in the range of hundreds of nanometers to several micrometers. ${ }^{28}$ The dominant scattering of these phonons is therefore by the amorphous surface of the NW. Recent works show that in NWs with rough or amorphous surfaces, Anderson localization could take place. ${ }^{34,35}$ The effect was shown to be increasingly important for thinner wires. ${ }^{20}$ Another cause could be the coupling of the localized modes in the amorphous oxide layer to propagating modes in the crystalline core. ${ }^{36,37}$ Such coupling could result in an effective layer near the boundary of the wire where the thermal conductivity is greatly reduced, an effect that can be much stronger for thinner wires. Finally, within the hydrodynamic framework, ${ }^{3-5,10}$ the so-called no-slip condition could also lead to a strong reduction of the thermal conductivity near the boundary of the NW, quenching the contribution to the heat flow of diffusive phonons. A possible origin for the enhancement of the contribution of specular phonons and the emergence of ballisticity is a strongly reduced scattering of these phonons into diffusive modes caused by localization-induced decoupling of specular and diffusive phonons. This scenario, which would agree with the observed weak $T$ dependence of the transition, is explained in Section SI10.

These qualitative explanations of the reduction of the contribution of diffusive phonons to the heat flow and the enhancement of the contribution of specular phonons should be quantified, such that they can be experimentally verified. Ballistic phonon transport may find application in phonon transistors, ${ }^{38,39}$ phonon waveguides, ${ }^{40}$ and in novel cooling solutions for computer chips, which require rapid removal of heat from ever decreasing volumes. Ballistic heat flow could possibly be realized more easily in other compound semiconductors composed of lighter elements than Ga and P, such as $\mathrm{Al}$ and $\mathrm{N}$, because of even longer phonon MFPs.

\section{ASSOCIATED CONTENT}

\section{Supporting Information}

The Supporting Information is available free of charge at https://pubs.acs.org/doi/10.1021/acs.nanolett.0c00320.

Nanowire growth, elemental analysis, thermal conductance measurements, background conductance, diffusive heat flow in $75 \mathrm{~nm}$ diameter nanowires, Raman thermometry, application of Landauer's formalism for heat flow, calculation of the numbers of specular and diffusive phonon modes, contributions of specular and diffusive phonons to thermal conductivity, decoupling of specular and localized diffusive phonons (PDF)

\section{AUTHOR INFORMATION}

\section{Corresponding Author}

Erik P. A. M. Bakkers - Department of Applied Physics, Eindhoven University of Technology, 5600 MB Eindhoven, The Netherlands; 10 orcid.org/0000-0002-8264-6862; Email: e.p.a.m.bakkers@tue.nl

\section{Authors}

Daniel Vakulov - Department of Applied Physics, Eindhoven University of Technology, 5600 MB Eindhoven, The Netherlands

Subash Gireesan - Department of Applied Physics, Eindhoven University of Technology, 5600 MB Eindhoven, The Netherlands; Center for Computational Energy Research, 5600 HH Eindhoven, The Netherlands

Milo Y. Swinkels - Department of Physics, University of Basel, 4056 Basel, Switzerland

Ruben Chavez - Department of Applied Physics, Eindhoven University of Technology, 5600 MB Eindhoven, The Netherlands

Tom Vogelaar - Department of Applied Physics, Eindhoven University of Technology, 5600 MB Eindhoven, The Netherlands

Pol Torres - Departament de Fisica, Universitat Autònoma de Barcelona, Bellaterra 08193, Spain

Alessio Campo - Department of Physics, University of Basel, 4056 Basel, Switzerland

Marta De Luca - Department of Physics, University of Basel, 4056 Basel, Switzerland; 다이.org/0000-0001-6061-3261

Marcel A. Verheijen - Department of Applied Physics, Eindhoven University of Technology, 5600 MB Eindhoven, The Netherlands

Sebastian Koelling - Department of Applied Physics, Eindhoven University of Technology, 5600 MB Eindhoven, The Netherlands 
Luca Gagliano - Department of Applied Physics, Eindhoven University of Technology, 5600 MB Eindhoven, The Netherlands; (1) orcid.org/0000-0003-4214-8639

Jos E. M. Haverkort - Department of Applied Physics, Eindhoven University of Technology, $5600 \mathrm{MB}$ Eindhoven, The Netherlands; (1) orcid.org/0000-0003-3051-673X

F. Xavier Alvarez - Departament de Fisica, Universitat Autonoma de Barcelona, Bellaterra 08193, Spain; (1) orcid.org/0000-0001-6746-2144

Peter A. Bobbert - Department of Applied Physics, Eindhoven University of Technology, 5600 MB Eindhoven, The Netherlands; Center for Computational Energy Research, 5600 HH Eindhoven, The Netherlands

Ilaria Zardo - Department of Physics, University of Basel, 4056 Basel, Switzerland; 다. orcid.org/0000-0002-8685-2305

Complete contact information is available at: https://pubs.acs.org/10.1021/acs.nanolett.0c00320

\section{Author Contributions \\ ${ }^{\perp}$ These authors contributed equally to this work. \\ Notes}

The authors declare no competing financial interest.

\section{ACKNOWLEDGMENTS}

We would like to acknowledge Rob van der Heijden, Riccardo Rurali, Michel de Jong, Bernd Gotsmann, Ali Shakouri, and Chris Palmstrøm for valuable feedback on the manuscript. This work has been supported by the European Research Council (ERC 617256 and 756365), the Dutch Organization for Scientific Research (NWO), the Foundation for Fundamental Research on Matter (FOM), and the Swiss National Science Foundation research grant (project grant no. 165784). We acknowledge Solliance, a solar energy $\mathrm{R} \& \mathrm{D}$ initiative of $\mathrm{ECN}$, TNO, Holst, TU/e, imec and Forschungszentrum Jülich, and the Dutch province of Noord-Brabant for funding the TEM facility. This work is part of the Industrial Partnership Programme (IPP) "Computational sciences for energy research" of The Netherlands Organization for Scientific Research Institutes (NWO-I). This research program is cofinanced by Shell Global Solutions International B.V. We acknowledge financial support by the Spain's Ministerio de Ciencia, Innovacion y Universidades under grant No. RTI2018-097876-B-C21 (MCIU/AEI/FEDER, UE).

\section{REFERENCES}

(1) Fourier, J. B. J. Théorie Analytique de La Chaleur; Didot, F., Ed.; Cambridge University Press: Cambridge, 1822.

(2) Maire, J.; Anufriev, R.; Yanagisawa, R.; Ramiere, A.; Volz, S.; Nomura, M. Heat Conduction Tuning by Wave Nature of Phonons. Sci. Adv. 2017, 3 (8), No. e1700027.

(3) Alvarez, F. X.; Jou, D.; Sellitto, A. Phonon Hydrodynamics and Phonon-Boundary Scattering in Nanosystems. J. Appl. Phys. 2009, 105 (1), 014317

(4) Cepellotti, A.; Fugallo, G.; Paulatto, L.; Lazzeri, M.; Mauri, F.; Marzari, N. Phonon Hydrodynamics in Two-Dimensional Materials. Nat. Commun. 2015, 6, 6400.

(5) Lee, S.; Broido, D.; Esfarjani, K.; Chen, G. Hydrodynamic Phonon Transport in Suspended Graphene. Nat. Commun. 2015, 6, 6290.

(6) Ding, Z.; Zhou, J.; Song, B.; Chiloyan, V.; Li, M.; Liu, T.-H.; Chen, G. Phonon Hydrodynamic Heat Conduction and Knudsen Minimum in Graphite. Nano Lett. 2018, 18 (1), 638-649.

(7) Torres, P.; Torelló, A.; Bafaluy, J.; Camacho, J.; Cartoixà, X.; Alvarez, F. X. First Principles Kinetic-Collective Thermal Con- ductivity of Semiconductors. Phys. Rev. B: Condens. Matter Mater. Phys. 2017, 95 (16), 165407.

(8) Ziabari, A.; Torres, P.; Vermeersch, B.; Xuan, Y.; Cartoixà, X.; Torelló, A.; Bahk, J.-H.; Koh, Y. R.; Parsa, M.; Peide, D. Y.; et al. FullField Thermal Imaging of Quasiballistic Crosstalk Reduction in Nanoscale Devices. Nat. Commun. 2018, 9 (1), 255.

(9) Martelli, V.; Jiménez, J. L.; Continentino, M.; Baggio-Saitovitch, E.; Behnia, K. Thermal Transport and Phonon Hydrodynamics in Strontium Titanate. Phys. Rev. Lett. 2018, 120 (12), 125901.

(10) Beardo, A.; Calvo-Schwarzwälder, M.; Camacho, J.; Myers, T. G.; Torres, P.; Sendra, L.; Alvarez, F. X.; Bafaluy, J. Hydrodynamic Heat Transport in Compact and Holey Silicon Thin Films. Phys. Rev. Appl. 2019, 11 (3), 34003.

(11) Siemens, M. E.; Li, Q.; Yang, R.; Nelson, K. A.; Anderson, E. H.; Murnane, M. M.; Kapteyn, H. C. Quasi-Ballistic Thermal Transport from Nanoscale Interfaces Observed Using Ultrafast Coherent Soft X-Ray Beams. Nat. Mater. 2010, 9 (1), 26-30.

(12) Bae, M.-H.; Li, Z.; Aksamija, Z.; Martin, P. N.; Xiong, F.; Ong, Z.-Y.; Knezevic, I.; Pop, E. Ballistic to Diffusive Crossover of Heat Flow in Graphene Ribbons. Nat. Commun. 2013, 4, 1734.

(13) Hsiao, T.-K.; Chang, H.-K.; Liou, S.-C.; Chu, M.-W.; Lee, S.-C.; Chang, C.-W. Observation of Room-Temperature Ballistic Thermal Conduction Persisting over $8.3 \$ \mu \$ \mathrm{~m}$ in SiGe Nanowires. Nat. Nanotechnol. 2013, 8 (7), 534.

(14) Wilson, R. B.; Cahill, D. G. Anisotropic Failure of Fourier Theory in Time-Domain Thermoreflectance Experiments. Nat. Commun. 2014, 5, 5075.

(15) Lee, J.; Lim, J.; Yang, P. Ballistic Phonon Transport in Holey Silicon. Nano Lett. 2015, 15 (5), 3273-3279.

(16) Hu, Y.; Zeng, L.; Minnich, A. J.; Dresselhaus, M. S.; Chen, G. Spectral Mapping of Thermal Conductivity through Nanoscale Ballistic Transport. Nat. Nanotechnol. 2015, 10 (8), 701.

(17) Anufriev, R.; Ramiere, A.; Maire, J.; Nomura, M. Heat Guiding and Focusing Using Ballistic Phonon Transport in Phononic Nanostructures. Nat. Commun. 2017, 8, 15505.

(18) Anufriev, R.; Gluchko, S.; Volz, S.; Nomura, M. Quasi-Ballistic Heat Conduction Due to Lévy Phonon Flights in Silicon Nanowires. ACS Nano 2018, 12, 11928.

(19) Hicks, L. D.; Dresselhaus, M. S. Thermoelectric Figure of Merit of a One-Dimensional Conductor. Phys. Rev. B: Condens. Matter Mater. Phys. 1993, 47 (24), 16631.

(20) Murphy, P. G.; Moore, J. E. Coherent Phonon Scattering Effects on Thermal Transport in Thin Semiconductor Nanowires. Phys. Rev. B: Condens. Matter Mater. Phys. 2007, 76 (15), 155313.

(21) Wen, Y.-C.; Hsieh, C.-L.; Lin, K.-H.; Chen, H.-P.; Chin, S.-C.; Hsiao, C.-L.; Lin, Y.-T.; Chang, C.-S.; Chang, Y.-C.; Tu, L.-W.; et al. Specular Scattering Probability of Acoustic Phonons in Atomically Flat Interfaces. Phys. Rev. Lett. 2009, 103 (26), 264301.

(22) Bas, P.; Bostel, A.; Deconihout, B.; Blavette, D. A General Protocol for the Reconstruction of 3D Atom Probe Data. Appl. Surf. Sci. 1995, 87, 298-304.

(23) Shi, L.; Li, D.; Yu, C.; Jang, W.; Kim, D.; Yao, Z.; Kim, P.; Majumdar, A. Measuring Thermal and Thermoelectric Properties of One-Dimensional Nanostructures Using a Microfabricated Device. J. Heat Transfer 2003, 125 (5), 881-888.

(24) Swinkels, M. Y.; van Delft, M. R.; Oliveira, D. S.; Cavalli, A.; Zardo, I.; van der Heijden, R. W.; Bakkers, E. P. A. M. Diameter Dependence of the Thermal Conductivity of InAs Nanowires. Nanotechnology 2015, 26 (38), 385401.

(25) Levinshtein, M.; Rumyantsev, S.; Shur, M. Handbook Series on Semiconductor Parameters; World Scientific: Singapore, 1996.

(26) Ordonez-Miranda, J.; Tranchant, L.; Kim, B.; Chalopin, Y.; Antoni, T.; Volz, S. Quantized Thermal Conductance of Nanowires at Room Temperature Due to Zenneck Surface-Phonon Polaritons. Phys. Rev. Lett. 2014, 112 (5), 55901.

(27) Haynes, W. M. CRC Handbook of Chemistry and Physics; CRC Press: Boca Raton, FL, 2014.

(28) Gireesan, S.; Torres, P.; Alvarez, F. X.; Bobbert, P. A. DiameterDependent Thermal Conductivity of Ultrathin GaP Nanowires: A 
Molecular Dynamics Study. Phys. Rev. B: Condens. Matter Mater. Phys. 2020, 101, 024307.

(29) Olsson, K. S.; An, K.; Li, X. Magnon and Phonon Thermometry with Inelastic Light Scattering. J. Phys. D: Appl. Phys. 2018, 51 (13), 133001.

(30) Maassen, J.; Lundstrom, M. Steady-State Heat Transport: Ballistic-to-Diffusive with Fourier's Law. J. Appl. Phys. 2015, 117 (3), 035104.

(31) de Picciotto, R.; Stormer, H. L.; Pfeiffer, L. N.; Baldwin, K. W.; West, K. W. Four-Terminal Resistance of a Ballistic Quantum Wire. Nature 2001, 411 (6833), 51.

(32) Chen, R.; Hochbaum, A. I.; Murphy, P.; Moore, J.; Yang, P.; Majumdar, A. Thermal Conductance of Thin Silicon Nanowires. Phys. Rev. Lett. 2008, 101 (10), 105501.

(33) Anderson, P. W. Absence of Diffusion in Certain Random Lattices. Phys. Rev. 1958, 109 (5), 1492.

(34) Xiong, S.; Sääskilahti, K.; Kosevich, Y. A.; Han, H.; Donadio, D.; Volz, S. Blocking Phonon Transport by Structural Resonances in Alloy-Based Nanophononic Metamaterials Leads to Ultralow Thermal Conductivity. Phys. Rev. Lett. 2016, 117 (2), 25503.

(35) Markoš, P.; Muttalib, K. A. Phonon Localization in Nanowires Dominated by Surface Roughness. Phys. Rev. B: Condens. Matter Mater. Phys. 2019, 99 (13), 134208.

(36) Xiong, S.; Selli, D.; Neogi, S.; Donadio, D. Native Surface Oxide Turns Alloyed Silicon Membranes into Nanophononic Metamaterials with Ultralow Thermal Conductivity. Phys. Rev. B: Condens. Matter Mater. Phys. 2017, 95 (18), 180301.

(37) Verdier, M.; Han, Y.; Lacroix, D.; Chapuis, P.-O.; Termentzidis, K. Radial Dependence of Thermal Transport in Silicon Nanowires. J. Phys. Mater. 2019, 2 (1), 015002.

(38) Wang, L.; Li, B. Thermal Logic Gates: Computation with Phonons. Phys. Rev. Lett. 2007, 99 (17), 177208.

(39) Sklan, S.; Splash, R. Pop, Sizzle: Information Processing with Phononic Computing. AIP Adv. 2015, 5 (5), 053302.

(40) Hatanaka, D.; Mahboob, I.; Onomitsu, K.; Yamaguchi, H. Phonon Waveguides for Electromechanical Circuits. Nat. Nanotechnol. 2014, 9 (7), 520.

\section{NOTE ADDED AFTER ASAP PUBLICATION}

Due to a production error, this paper was published ASAP on March, 3, 2020, with an incorrect version of Figure 2. The corrected version was reposted on March 5, 2020. 